Research Article

\title{
Stability of Moving Mass Control Spinning Missiles with Angular Rate Loops
}

\author{
Qiushi Zheng (i) and Zhiming Zhou \\ Beijing Institute of Technology, 10081 Beijing, China \\ Correspondence should be addressed to Qiushi Zheng; zhengqiushi1993@163.com
}

Received 27 June 2019; Revised 6 September 2019; Accepted 8 October 2019; Published 27 November 2019

Academic Editor: Andrzej Swierniak

Copyright (c) 2019 Qiushi Zheng and Zhiming Zhou. This is an open access article distributed under the Creative Commons Attribution License, which permits unrestricted use, distribution, and reproduction in any medium, provided the original work is properly cited.

\begin{abstract}
Moving mass control (MMC) is a new control method in control field. It is a potential way to solve the problem of aerodynamic rudder control insufficiency caused by the low density of upper atmosphere, to reduce the high speed missile aerodynamic thermal load, and to solve the problem of rudder surface ablation. However, the spinning of the airframe and the movement of internal moving mass induce the serious dynamic cross-coupling between pitch and yaw channels, which may lead to system instability in the form of a divergent coning motion. In this paper, the mathematical model of the MMC missile is established, and the angular motion equation is finally obtained by some linearized assumptions. Then, the sufficient and necessary conditions of coning motion stability for MMC missiles with angular rate loops under fast and slow spinning rates are analytically derived and further verified by numerical simulations. It is noticed that the upper bound of the control gain is affected by the location of the moving mass and the spinning rate of the missile.
\end{abstract}

\section{Introduction}

The moving mass control (MMC) technology changes the position of center of mass of the system by the displacement of the internal moving mass to generate corresponding control torques, thereby changing the flight attitude of the missile [1-3]. Moving mass control missile has attracted much attention because of its special advantages. When the missile flies in the high altitude, the conventional aerodynamic control cannot provide the required lateral acceleration, as the density of air is too low. However, the moving mass control has the potential to solve this problem. Moreover, as the moving mass is arranged in the airframe, the missile has a better aerodynamic property, which reduces the aerodynamic heat of the warhead and avoids the problem of rudder ablation [2]. According to the number of moving masses of the actuator, the MMC missiles can be divided into three types: single MMC missiles [4], double MMC missiles [5], and triple MMC missiles.

There is a heavy coupling between the pitch and yaw channel of the moving mass control spinning missile. On the one hand, it is due to Magnus and gyroscopic effects caused by the rotation of the missile. On the other hand, the motion of the moving mass causes the deviation of the center of mass of the system and the deviation of the principal axis of inertia, which aggravates the coupling between pitch and yaw channels. Many studies have been proposed focusing on the control for such a system with heavy coupling, nonlinear dynamics, and parametric uncertainties. Zhang et al. [6] divided the dynamics of the MMAV into the fast state part and the slow state part and designed an autopilot for a nonlinear 6-DoF mass moment aerospace vehicle based on fuzzy sliding mode control, using dynamic inversion techniques. Then, Zhang et al. [7] designed the flight control system for the MMAV via utilizing nonlinear predictive control approach.

As for the stability for spinning missiles, many theoretical research studies have already been proposed. Murphy and Flatus [8-10] analyzed the factors that cause the coning motion of the missile, including the Magnus effect, inertial gyroscope effect, and aerodynamic asymmetry. Furthermore, for the stability of controlled spinning missiles, Yan et al. [11] studied the stability conditions of spinning missiles 
with rate loop, Li et al. [12] studied the stability of spinning missiles with an acceleration autopilot. In addition, Zhou et al. [13] studied the coning motion instability induced by hinge moment of the actuator.

Previous research studies mainly focus on aerodynamic control missiles. For the study of the stability of spinning aircraft with internal moving masses, current research studies are mostly focused on the instability of coning motion induced by mass deviation. For example, Carrier and Miles [14] proposed that the center of mass of the rocket changes due to the internal fluid motion, which led to unstable coning motion of the rocket. El-Gohary $[15,16]$ studied the stability of the mass moment satellite by means of the Lyapunov equation and obtained the required force and torque of the servo system satisfying the stability conditions. However, few of the existing literatures have considered the coning motion of a moving mass control spinning missile with the control loop.

Thus, this paper focuses on the stability of coning motion for a double moving masses control spinning missile with angular rate loops. The mathematical model of the missile system is established. The sufficient and necessary condition of coning motion stability for MMC spinning missiles with angular rate loops is analytically derived and further verified by numerical simulations. The stability boundary of control gains is obtained, and moreover, the influence of installation position of moving masses and spinning rate of the missile on stability is analyzed.

\section{System Configuration}

The moving mass control spinning missile proposed in this paper consists of a rigid body $B$ and two radial internal moving masses $m_{1}$ and $m_{2}$ as shown in Figure 1. The moving mass $m_{1}$ moves along the $y_{b}$ axis while the moving mass $m_{2}$ moves along the $z_{b}$ axis. The mass of the body $B$ is $m_{b}$, and the two moving masses $m_{1}$ and $m_{2}$ have the same mass $m$; thus, the total mass of the missile system is $m_{s}=m_{b}+2 m$. The mass ratio of the moving mass is $\mu=m / m_{s}$. $l$ is the installation position of the moving mass, and $\delta_{y}$ and $\delta_{z}$ are the radial displacements of the two moving masses in the nonspinning coordinate system.

\section{Mathematical Model}

The missile system is composed of three parts: the projectile body $\mathrm{B}$ and two moving masses. According to the momentum theorem of particle system, the translational dynamics of the missile system can be described as

$$
m_{\mathrm{B}} \frac{\mathrm{d} \mathbf{V}_{\mathrm{B}}}{\mathrm{d} t}+\sum_{i=1}^{2} m_{i} \frac{\mathrm{d} \mathbf{V}_{i}}{\mathrm{~d} t}=\mathbf{F}+L(\vartheta, \psi) m_{s} \mathbf{g}
$$

where $\mathbf{V}_{\mathrm{B}}$ is the velocity vector of the body $\mathrm{B}$ relative to the center of mass of the missile system $S^{*}$ and is given by

$$
\mathbf{V}_{\mathrm{B}}=\mathbf{V}-\gamma \times\left(\mathbf{r}_{1}+\mathbf{r}_{2}\right),
$$

where $\mathbf{V}=\left[\begin{array}{lll}u & v & w\end{array}\right]^{T}$ is the velocity vector of the body $\mathrm{B}$ in the nonspinning coordinate system, $\gamma=\left[\begin{array}{lll}\dot{\gamma} & 0 & 0\end{array}\right]^{T}$ is the

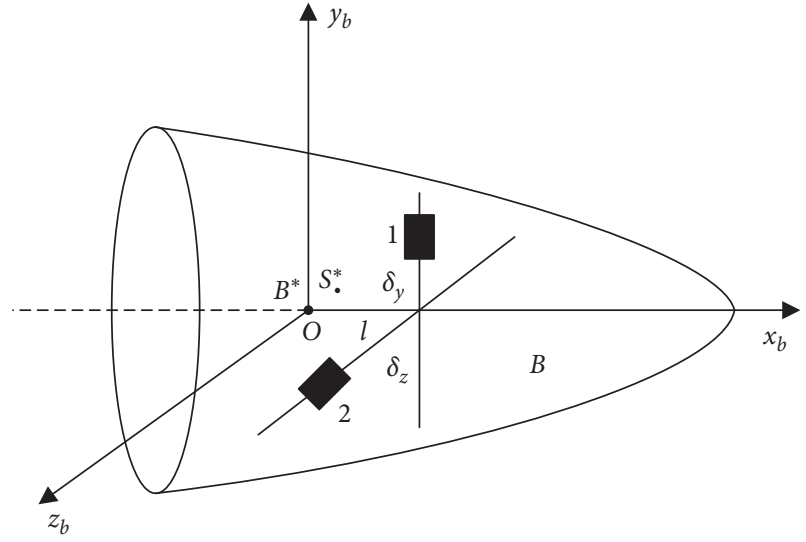

FIGURE 1: System configuration of the moving mass control missile.

spinning velocity vector, and $\mathbf{r}_{1}$ and $\mathbf{r}_{2}$ are position vectors of the two moving masses in the nonspinning CS. The derivative of equation (2) can be derived as

$$
\begin{aligned}
\frac{\mathrm{d} \mathbf{V}_{\mathrm{B}}}{\mathrm{d} t}= & \mathbf{V}+\omega_{4} \times \mathbf{V}-\left(\gamma \times\left(\mathbf{r}_{1}+\mathbf{r}_{2}\right)+\gamma \times\left(\mathbf{r}_{1}+\mathbf{r}_{2}\right)\right. \\
& \left.+\omega_{4} \times \gamma \times\left(\mathbf{r}_{1}+\mathbf{r}_{2}\right)\right),
\end{aligned}
$$

where $\omega_{4}$ is the angular rate of the nonspinning CS with respect to the inertial CS. The position vectors of the two masses in the nonspinning CS can be denoted as

$$
\begin{aligned}
& \mathbf{r}_{1}=\left[\begin{array}{lll}
l & \delta_{y 4} & 0
\end{array}\right]^{T}, \\
& \mathbf{r}_{2}=\left[\begin{array}{lll}
l & 0 & \delta_{z 4}
\end{array}\right]^{T},
\end{aligned}
$$

where $\delta_{y 4}$ and $\delta_{z 4}$ are projections of $\delta_{y}$ and $\delta_{z}$ on the nonspinning CS and are given by

$$
\left[\begin{array}{l}
\delta_{y 4} \\
\delta_{z 4}
\end{array}\right]=\left[\begin{array}{l}
\delta_{y} \cos \gamma-\delta_{z} \sin \gamma \\
\delta_{y} \sin \gamma+\delta_{z} \cos \gamma
\end{array}\right] .
$$

The velocity vector of each moving mass relative to the center of mass of the missile system $S^{*}$ can be expressed as

$$
\mathbf{V}_{i}=\mathbf{V}+\frac{\mathrm{d} \mathbf{r}_{i}}{\mathrm{~d} t}+\omega_{4} \times \mathbf{r}_{i} .
$$

The derivative of equation (6) can be derived as

$\frac{\mathrm{d} \mathbf{V}_{i}}{\mathrm{~d} t}=\mathbf{V}+\omega_{4} \times \mathbf{V}+\mathbf{r}_{i}+\omega_{4} \times \mathbf{r}_{i}+2 \times \omega_{4} \times \mathbf{r}_{i}+\omega_{4} \times\left(\omega_{4} \times \mathbf{r}_{i}\right)$.

Substituting equations (3) and (7) into equation (1) yields

$$
\begin{aligned}
& \sum_{i=1}^{2} m_{i}\left(\mathbf{r}_{i}+\omega_{4} \times \mathbf{r}_{i}+2 \times \omega_{4} \times \mathbf{r}_{i}+\omega_{4} \times\left(\omega_{4} \times \mathbf{r}_{i}\right)\right) \\
& \quad-m_{\mathrm{B}}\left(\gamma \times\left(\mathbf{r}_{1}+\mathbf{r}_{2}\right)+\gamma \times\left(\mathbf{r}_{1}+\mathbf{r}_{2}\right)+\omega_{4} \times \gamma \times\left(\mathbf{r}_{1}+\mathbf{r}_{2}\right)\right) \\
& \quad+m_{s}\left(\mathbf{V}+\omega_{4} \times \mathbf{V}\right)=\mathbf{F}+L(\vartheta, \psi) m_{s} \mathbf{g},
\end{aligned}
$$

where $\mathbf{F}$ is the vector of aerodynamic force in the nonspinning $\mathrm{CS}$ and is given by 


$$
\mathbf{F}=\left[\begin{array}{c}
-X \\
Y \\
Z
\end{array}\right]=Q S\left[\begin{array}{c}
-C_{x} \\
C_{y}^{\alpha} \alpha \\
-C_{y}^{\alpha} \beta
\end{array}\right]
$$

According to the theorem of angular momentum, the rotational motion of the missile system can be described as

$$
\frac{\mathrm{d} \mathbf{H}_{\mathrm{B}}}{\mathrm{d} t}+\sum_{i=1}^{2} \frac{\mathrm{d} \mathbf{H}_{i}}{\mathrm{~d} t}=\mathbf{M}_{S^{*}},
$$

where $\mathbf{H}_{\mathrm{B}}$ is the angular momentum of the body $\mathrm{B}, \mathbf{H}_{i}$ is the angular momentum of the moving mass, and $\mathbf{M}_{S^{*}}$ is the external moments applied on the missile system, including aerodynamic moments and mass eccentricity moments. $\mathbf{H}_{B}$, $\mathbf{H}_{i}$, and $\mathbf{M}_{S^{*}}$ are given by

$$
\begin{aligned}
& \mathbf{H}_{\mathrm{B}}=\mathbf{I}_{\mathrm{B}} \omega_{1}+\left(\mu_{1}+\mu_{2}\right)^{2} m_{\mathrm{B}} \sum_{i=1}^{2} \mathbf{r}_{i} \times\left(\mathbf{V}_{1}+\mathbf{V}_{2}\right), \\
& \mathbf{H}_{i}=\left(1-\sum_{i=1}^{2} \mu_{i}\right)^{2} m_{i} \mathbf{r}_{i} \times\left(\mathbf{V}_{1}+\mathbf{V}_{2}\right),
\end{aligned}
$$

$$
\mathbf{M}_{S^{*}}=\mathbf{M}-\sum_{i=1}^{2} \mu_{i} \mathbf{r}_{i} \times \mathbf{F}_{n b},
$$

where $\omega_{1}$ is the angular rate of the body CS with respect to the inertial CS. Substituting equations (10)-(12) into equation (9) yields

$$
\begin{aligned}
& \mathbf{I}_{\mathrm{B}} \omega_{1}+\omega_{4} \times \mathbf{I}_{\mathrm{B}} \omega_{1}+m_{\mathrm{B}}\left(\sum_{i=1}^{2} \mu_{i} \mathbf{r}_{i} \times\left(\mathbf{V}_{1}+\mathbf{V}_{2}\right)\right. \\
& \left.+\omega_{4} \times \sum_{i=1}^{2} \mu_{i} \mathbf{r}_{i} \times\left(\mathbf{V}_{1}+\mathbf{V}_{2}\right)\right)=\mathbf{M}-\sum_{i=1}^{2} \mu_{i} \mathbf{r}_{i} \times \mathbf{F}_{n b}
\end{aligned}
$$

The moments applied on the missile in the nonspinning CS are given by

$$
\mathbf{M}=\left[\begin{array}{c}
M_{x} \\
M_{y} \\
M_{z}
\end{array}\right]=Q S L\left[\begin{array}{c}
m_{\omega x}^{\prime}(L / V) \omega_{x 4} \\
m_{y}^{\alpha} \beta+m_{\omega y}^{\prime}(L / V) \omega_{y 4}-m_{\mu} \dot{\gamma} \alpha \\
m_{y}^{\alpha} \alpha+m_{\omega y}^{\prime}(L / V) \omega_{z 4}+m_{\mu} \dot{\gamma} \beta
\end{array}\right] .
$$

By substituting equation (9) into equation (8) and equation (15) into equation (14), the dynamic equations of the missile system can be finally obtained as

$$
\begin{gathered}
{\left[\begin{array}{c}
\dot{u} \\
\dot{v} \\
\dot{w}
\end{array}\right]+\left[\omega_{4}\right]\left[\begin{array}{c}
u \\
v \\
w
\end{array}\right]+\left[\begin{array}{cc}
0 & 0 \\
\mu_{1} & 0 \\
0 & \mu_{2}
\end{array}\right]\left[\begin{array}{l}
\ddot{\delta}_{y 4} \\
\ddot{\delta}_{z 4}
\end{array}\right]+[\mathbf{r}]\left[\begin{array}{c}
\dot{\omega}_{x 4} \\
\dot{\omega}_{y 4} \\
\dot{\omega}_{z 4}
\end{array}\right]+[\mathbf{r}]\left[\begin{array}{c}
2 \omega_{x 4}-\frac{m_{\mathrm{B}}}{m_{s} \dot{\gamma}} \\
2 \omega_{y 4} \\
2 \omega_{z 4}
\end{array}\right]+\left(\left[\omega^{2}\right]-\frac{m_{\mathrm{B}}}{m_{s}}\left[\omega_{4}\right][\gamma]\right)\left[\begin{array}{c}
\left(\mu_{1}+\mu_{2}\right) l \\
\delta_{y 4} \\
\delta_{z 4}
\end{array}\right]=\frac{1}{m_{s}}\left[\begin{array}{c}
-X \\
Y \\
Z
\end{array}\right]+L(\vartheta, \psi)\left[\begin{array}{c}
0 \\
g \\
0
\end{array}\right],} \\
{[\mathbf{I}]\left[\begin{array}{c}
\dot{\omega}_{x 4} \\
\dot{\omega}_{y 4} \\
\dot{\omega}_{z 4}
\end{array}\right]+m_{\mathrm{B}}\left[\begin{array}{cc}
-\mu_{2} \delta_{z 4} & \mu_{1} \delta_{y 4} \\
0 & -\left(\mu_{1}+\mu_{2}\right) l \\
\left(\mu_{1}+\mu_{2}\right) l & 0
\end{array}\right]\left[\begin{array}{l}
\ddot{\delta}_{y} \\
\ddot{\delta}_{z}
\end{array}\right]+\left[\begin{array}{c}
I_{x} \ddot{\gamma} \\
I_{x} \dot{\gamma} \omega_{z 4} \\
-I_{x} \dot{\gamma} \omega_{y 4}
\end{array}\right]+2 m_{B}[\mathbf{r}]\left[\omega_{4}\right]\left[\begin{array}{c}
0 \\
\dot{\delta}_{y 4} \\
\dot{\delta}_{z 4}
\end{array}\right]+\left[\omega^{2}\right][\mathbf{r}]\left[\begin{array}{c}
\left(\mu_{1}+\mu_{2}\right) l \\
\delta_{y 4} \\
\delta_{z 4}
\end{array}\right]=\left[\begin{array}{c}
M_{x} \\
M_{y} \\
M_{z}
\end{array}\right]-[\mathbf{r}]\left[\begin{array}{c}
-X \\
Y \\
Z
\end{array}\right],}
\end{gathered}
$$

where

$$
\begin{aligned}
& {\left[\omega_{4}\right]=\left[\begin{array}{ccc}
0 & -\omega_{z 4} & \omega_{y 4} \\
\omega_{z 4} & 0 & -\omega_{x 4} \\
-\omega_{y 4} & \omega_{x 4} & 0
\end{array}\right],} \\
& {[\dot{\gamma}]=\left[\begin{array}{lll}
0 & 0 & 0 \\
0 & 0 & \dot{\gamma} \\
0 & \dot{\gamma} & 0
\end{array}\right],} \\
& {\left[\omega^{2}\right]=\left[\omega_{4}\right]\left[\omega_{4}\right],}
\end{aligned}
$$




$$
\begin{aligned}
{\left[\mathbf{r}_{1}\right] } & =\left[\begin{array}{ccc}
0 & 0 & -\delta_{y 4} \\
0 & 0 & l \\
\delta_{y 4} & -l & 0
\end{array}\right], \\
{\left[\mathbf{r}_{2}\right] } & =\left[\begin{array}{ccc}
0 & \delta_{z 4} & 0 \\
-\delta_{z 4} & 0 & l \\
0 & -l & 0
\end{array}\right], \\
{[\mathbf{r}] } & =\mu_{1}\left[\mathbf{r}_{1}\right]+\mu_{2}\left[\mathbf{r}_{2}\right], \\
{[\mathbf{I}] } & =\left[\begin{array}{ccc}
I_{x}+m_{B}\left(\mu_{1} \delta_{y 4}^{2}+\mu_{2} \delta_{z 4}^{2}\right) & -m_{B}\left(\mu_{1}+\mu_{2}\right) l \delta_{y 4} & -m_{B}\left(\mu_{1}+\mu_{2}\right) l \delta_{z 4} \\
-m_{B}\left(\mu_{1}+\mu_{2}\right) l \delta_{y 4} & I_{y}+m_{B}\left(\left(\mu_{1}+\mu_{2}\right)^{2} l^{2}+\mu_{2} \delta_{z 4}^{2}\right) & -m_{B} \mu_{1} \delta_{y 4} \delta_{z 4} \\
-m_{B}\left(\mu_{1}+\mu_{2}\right) l \delta_{z 4} & -m_{B} \mu_{2} \delta_{y 4} \delta_{z 4} & I_{z}+m_{B}\left(\mu_{1} \delta_{y 4}^{2}+\left(\mu_{1}+\mu_{2}\right)^{2} l^{2}\right)
\end{array}\right] .
\end{aligned}
$$

\section{Angular Motion of the Moving Mass Control Spinning Missile}

Even though the mathematical model described in equations (16) and (17) is more accurate and close to the real case, due to the highly nonlinear equations of motion, it is difficult to get the analytical solution and the obvious relationship between the flight characteristics of the missile and control parameters. To facilitate theoretical analysis, the general method is to apply the linearization theory of projectile. This theory has been regarded as an effective tool to analyze the flight stability of projectiles and applied in references [8-13]. Therefore, in order to linearize these two equations, the following assumptions are introduced:

(1) The mass ratio is small, so $\mu=\mu_{1}=\mu_{2} \ll 1,1-\mu \approx 1$

(2) The spinning rate in the nonspinning CS $\omega_{x 4}$ keeps constant and is equal to zero, and $\ddot{\gamma}$ is small, so $\ddot{\gamma}=0$

(3) Variables $\omega_{y 4}, \omega_{z 4}, v, w, \alpha$, and $\beta$ are small

(4) The gravity effect is negligible

(5) $l$ keeps constant, so $\dot{l}=\ddot{l}=0$

(6) The missile is strictly axisymmetric, so $I_{y}=I_{z}$

Under these assumptions, the equations for lateral translational and rotational motion in equations (16) and (17) can be simplified to

$$
\begin{aligned}
& \left\{\begin{array}{l}
\dot{v}=\frac{Y}{m_{s}}-\omega_{z 4} u-\left(\frac{m_{\mathrm{B}}}{m_{s}}\right) \dot{\gamma} \dot{\delta}_{z 4}, \\
\dot{w}=\frac{Z}{m_{s}}+\omega_{y 4} u+\left(\frac{m_{\mathrm{B}}}{m_{s}}\right) \dot{\gamma} \dot{\delta}_{y 4},
\end{array}\right. \\
& \left\{\begin{array}{l}
\dot{\omega}_{y 4}=\frac{M_{y}}{I_{2}}+\frac{2 \mu l Z}{I_{2}}+\frac{\mu X \delta_{z 4}}{I_{2}}-\left(\frac{I_{1}}{I_{2}}\right) \omega_{z 4} \dot{\gamma}+\frac{2 \mu m_{\mathrm{B}} l \ddot{\delta}_{z}}{I_{2}}, \\
\dot{\omega}_{z 4}=\frac{M_{z}}{I_{2}}-\frac{2 \mu l Y}{I_{2}}-\frac{\mu X \delta_{y 4}}{I_{2}}+\left(\frac{I_{1}}{I_{2}}\right) \omega_{y 4} \dot{\gamma}-\frac{2 \mu m_{\mathrm{B}} l \ddot{\delta}_{y}}{I_{2}} .
\end{array}\right.
\end{aligned}
$$

The angles of attack $\alpha$ and sideslip $\beta$ are defined as

$$
\left\{\begin{array}{l}
\alpha=-\arctan \left(\frac{v}{u}\right) \cong-\frac{v}{u}, \\
\beta=\arcsin \left(\frac{w}{V}\right) \cong \frac{w}{V} .
\end{array}\right.
$$

By defining the complex angle of attack $\xi=-\beta+i \alpha$, the complex angular rate $\Omega=\omega_{z 4}+i \omega_{y 4}$, and the complex control instruction $\delta=\delta_{y 4}-i \delta_{z 4}$, equation (19) can be reformulated as

$$
\Omega=-i \dot{\xi}+i \frac{Q S\left(C_{x}-C_{y}^{\alpha}\right)}{m_{s} V} \xi-i \frac{m_{\mathrm{B}} \dot{\gamma}}{m_{s} V} \dot{\delta} .
$$

Equation (20) can be reformulated as

$$
\begin{aligned}
\dot{\Omega}= & -i \frac{I_{1}}{I_{2}} \dot{\gamma} \Omega-\frac{2 \mu m_{\mathrm{B}} l}{I_{2}} \ddot{\delta}+i \frac{2 \mu l Q S C_{y}^{\alpha}}{I_{2}} \xi-i \frac{Q_{S L m_{y}^{\alpha}}^{\alpha}}{I_{2}} \xi \\
& -\frac{\mu Q S C_{x}}{I_{2}} \delta+\frac{Q S L m_{\omega y}^{\prime}}{I_{2}} \frac{L}{V} \Omega-\frac{Q S L m_{\mu} \dot{\gamma}}{I_{2}} \frac{L}{V} \xi .
\end{aligned}
$$

Substituting equation (22) into equation (23), the angular motion equation of the moving mass spinning missile can be obtained as

$$
\ddot{\xi}+A \dot{\xi}+B \xi=C,
$$

where $A=-m_{n}^{\omega}-k_{5}+i k_{1}, B=-m_{n}^{\alpha}+k_{4}+i\left(m_{m \alpha}+k_{1} k_{5}\right)$, $C=-i m_{n}^{\delta} \delta-\left(-m_{n}^{\omega} k_{3}+i k_{1} k_{3}\right) \dot{\delta}-\left(k_{3}+i k_{2}\right) \ddot{\delta}, k_{1}=\left(I_{1} / I_{2}\right) \dot{\gamma}$, $k_{2}=\left(2 \mu m_{\mathrm{B}} l\right) / I_{2}, \quad k_{3}=m_{\mathrm{B}} \dot{\gamma} / m_{s} V, k_{4}=\left(2 \mu l Q S C_{y}^{\alpha}\right) / I_{2}, \quad k_{5}=$ $\left(Q S\left(C_{x}-C_{y}^{\alpha}\right)\right) / m_{S} V, m_{n}^{\omega}=\left(Q S L^{2} C_{\omega z}^{\prime}\right) / I_{2}, m_{n}^{\alpha}=\left(Q S L m_{y}^{\alpha}\right)$ $/ I_{2}, m_{m \alpha}=\left(Q S L^{2} m_{\mu} \dot{\gamma}\right) / I_{2} V$, and $m_{n}^{\delta}=\left(Q S C_{x} \mu\right) / I_{2}$.

According equation (24), the equilibrium point is determined by

$$
\xi_{e}=\frac{C}{B}=\frac{C_{1}+C_{2}}{B}=\xi_{e 1}+\xi_{e 2},
$$

where $C_{1}=-i m_{n}^{\delta} \delta$ and $C_{2}=-\left(k_{1} k_{3}+i \dot{k_{3}}\right) \dot{\delta}-\left(k_{3}+i k_{2}\right) \ddot{\delta}$. 
$\xi_{e 1}$ is the complex angle of attack generated by system centroid offset caused by the movement of the moving mass. Suppose that the spinning rate of the missile is zero and the position of the moving mass remains fixed, we get

$$
\xi_{e 1}=\frac{-i m_{n}^{\delta} \delta}{-m_{n}^{\alpha}+k_{4}} .
$$

$\xi_{e 2}$ is the complex angle of attack generated by the offset of the principal axis of inertia and was estimated by Hodapp and Clark in [17] as

$$
\left|\xi_{e 2}\right| \cong \frac{\mu m_{S} l}{I_{2}} \delta_{\max }
$$

\section{Stability of the Moving Mass Spinning Missile with the Angular Rate Loop}

The control system with angular rate loops is shown in Figure 2, in which $n_{y}$ and $n_{z}$ are control commands, $\dot{\vartheta}$ and $\dot{\psi}$ are feedback signals, and $k_{\omega}$ is the gain.

It can be seen from Figure 2 that the input commands to the actuators can be described as

$$
\left[\begin{array}{l}
n_{y} \\
n_{z}
\end{array}\right]=\left[\begin{array}{cc}
-k_{\omega} & 0 \\
0 & -k_{\omega}
\end{array}\right]\left[\begin{array}{l}
\dot{\vartheta} \\
\dot{\psi}
\end{array}\right] \text {. }
$$

According to the definition of coordinate system and angle, negative angle of attack will generate positive pitching acceleration, while positive angle of sideslip will generate positive yaw acceleration. Therefore, the displacement instruction of the moving mass is obtained as

$$
\left[\begin{array}{l}
\delta_{y c} \\
\delta_{z c}
\end{array}\right]=\left[\begin{array}{cc}
-1 & 0 \\
0 & 1
\end{array}\right]\left[\begin{array}{l}
n_{y} \\
n_{z}
\end{array}\right] .
$$

Meanwhile, based on the assumption that the missile is in horizontal flight, there exists an approximation relationship: $\dot{\alpha}=\dot{\vartheta}$ and $\dot{\beta}=\dot{\psi}$. Thus, equation (29) can be expressed as

$$
\left[\begin{array}{l}
\delta_{y c} \\
\delta_{z c}
\end{array}\right]=\left[\begin{array}{cc}
-k_{\omega} & 0 \\
0 & k_{\omega}
\end{array}\right]\left[\begin{array}{l}
\dot{\alpha} \\
\dot{\beta}
\end{array}\right]
$$

Converting equation (30) into the complex form, one has

$$
\delta=i k_{\omega} \dot{\xi}
$$

Substituting equation (31) into equation (24) yields

$$
\begin{aligned}
& \left(-k_{2} k_{\omega}+i k_{3} k_{\omega}\right)+\left(1-k_{1} k_{3} k_{\omega}-i m_{n}^{\omega} k_{3} k_{\omega}\right) \ddot{\xi} \\
& \quad+\left(-k_{\omega} m_{n}^{\delta}-m_{n}^{\omega}-k_{5}+i k_{1}\right) \dot{\xi} \\
& \quad+\left(-m_{n}^{\alpha}+k_{4}+i\left(m_{m \alpha}+k_{1} k_{5}\right)\right) \xi=0 .
\end{aligned}
$$

5.1. Slow Spinning Rate Case. For slowly spinning missiles, the main factor for the generation of angle of attack is the mass eccentric moment caused by the movement of moving masses. Therefore, when studying the stability of slowly spinning missiles, the first- and second-order derivatives of

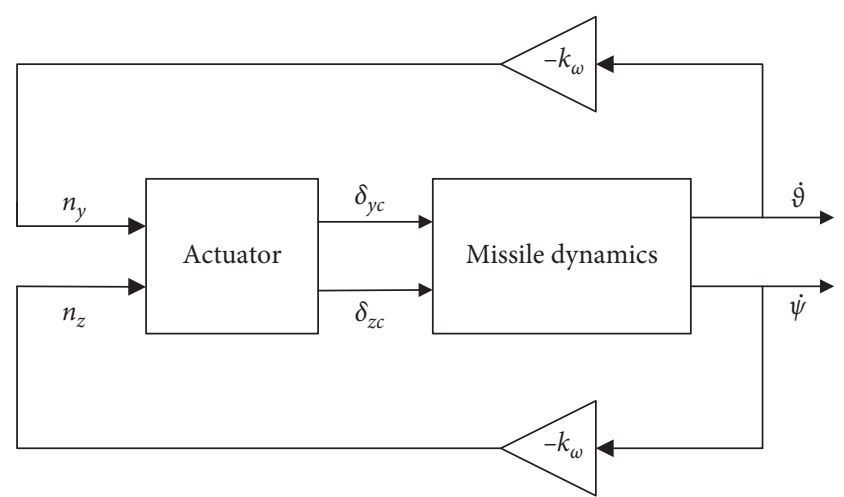

FIGURE 2: Moving mass control missile system with angular rate loops.

the position of moving masses can be ignored. Then, equation (32) can be simplified as

$$
\ddot{\xi}+\left(H_{c}+i P_{c}\right) \dot{\xi}-\left(M_{c}+i Q_{c}\right) \xi=0,
$$

where $H_{c}=-k_{\omega} m_{n}^{\delta}-m_{n}^{\omega}-k_{5}, P_{c}=k_{1}, M_{c}=m_{n}^{\alpha}-k_{4}$, and $Q_{c}=-\left(m_{m \alpha}+k_{1} k_{5}\right)$.

The corresponding characteristic equation is

$$
\lambda^{2}+\left(H_{c}+i P_{c}\right) \lambda-\left(M_{c}+i Q_{c}\right)=0 .
$$

Assuming $\left(H_{c}+i P_{c}\right)^{2}+4\left(M_{c}+i Q_{c}\right)=R_{c}$, where

$$
R_{c}=R_{\text {cre }}+i R_{\text {cim }} \text {, }
$$

one gets

$$
\left\{\begin{array}{l}
R_{c r e}=H_{c}^{2}-P_{c}^{2}+4 M_{c}, \\
R_{c i m}=2 H_{c} P_{c}+4 Q_{c},
\end{array}\right.
$$

Then, the characteristic roots of equation (34) are given by

$$
\lambda_{1,2}=\frac{1}{2}\left(-H_{c} \pm \sqrt{\frac{\left|R_{c}+R_{c r e}\right|}{2}}\right)+\frac{1}{2}\left(-P_{c} \pm \sqrt{\frac{\left|R_{c}\right|-R_{c r e}}{2}}\right) i .
$$

According to Lyapunov stability theory, the sufficient and necessary condition for stability of the moving mass missile under low spinning rate with rate loops can be obtained as

$$
-H_{c} \pm \sqrt{\frac{\left|R_{c}\right|+R_{\text {cre }}}{2}}<0 .
$$

Because $\sqrt{\left(\left|R_{c}\right|+R_{c r e}\right) / 2}>0$, in order to ensure that equation (38) is true, the following inequality must be met:

$$
-H_{c}<-\sqrt{\frac{\left|R_{c}\right|+R_{c r e}}{2}} .
$$

Substituting $H_{c}, R_{c}$, and $R_{c r e}$ into equation (39) yields

$$
\begin{aligned}
& \left(m_{n}^{\delta}\right)^{2} k_{\omega}^{2}\left(-m_{n}^{\alpha}+k_{4}\right)+\left(2\left(m_{n}^{\omega}+k_{5}\right)\left(-m_{n}^{\alpha}+k_{4}\right)\right. \\
& \left.\quad-k_{1}\left(m_{m \alpha}+k_{1} k_{5}\right)\right) m_{n}^{\delta} k_{\omega}+\left(m_{n}^{\omega}+k_{5}\right)^{2}\left(-m_{n}^{\alpha}+k_{4}\right) \\
& \quad-\left(m_{n}^{\omega}+k_{5}\right) k_{1}\left(m_{m \alpha}+k_{1} k_{5}\right)-\left(m_{m \alpha}+k_{1} k_{5}\right)^{2}>0 .
\end{aligned}
$$


To facilitate the analysis, a polynomial $f\left(k_{\omega}\right)$ is introduced:

$$
f\left(k_{\omega}\right)=a k_{\omega}^{2}+b k_{\omega}+c,
$$

where $a=\left(m_{n}^{\delta}\right)^{2}\left(-m_{n}^{\alpha}+k_{4}\right), b=\left(2\left(m_{n}^{\omega}+k_{5}\right)\left(-m_{n}^{\alpha}+k_{4}\right)-k_{1}\right.$ $\left.\left(m_{m \alpha}+k_{1} k_{5}\right)\right) m_{n}^{\delta}$, and $c=\left(m_{n}^{\omega}+k_{5}\right)^{2}\left(-m_{n}^{\alpha}+k_{4}\right)-\left(m_{n}^{\omega}+k_{5}\right)$ $k_{1}\left(m_{m \alpha}+k_{1} k_{5}\right)-\left(m_{m \alpha}+k_{1} k_{5}\right)^{2}$.

For slowly spinning missiles, $k_{1}$ and $m_{m \alpha}$ are small. The sign of $a$ and $b$ mainly depends on the sign of the first term on the right-hand side, so $a$ and $b$ have opposite signs. Two cases are discussed below:

(1) The first case is when $a>0$, one gets $-m_{n}^{\alpha}+k_{4}>0$, $b<0$, and $c>0$, and the curve of $f\left(k_{\omega}\right)$ is illustrated by Iin Figure 3 . The intersections of $f\left(k_{\omega}\right)$ and the axis are given by

$$
\left\{\begin{array}{l}
k_{\omega 11}=\frac{-b-\sqrt{b^{2}-4 a c}}{2 a} \\
k_{\omega 12}=\frac{-b+\sqrt{b^{2}-4 a c}}{2 a} .
\end{array}\right.
$$

Thus, only when $k_{\omega}<k_{\omega 11}$ or $k_{\omega}>k_{\omega 12}$, one gets $f\left(k_{\omega}\right)>0$. The sufficient and necessary condition for the coning motion stability can be derived as

$$
k_{\omega} \in\left(0,-\frac{m_{n}^{\omega}+k_{5}}{m_{n}^{\delta}}\right) \cap\left(0, k_{\omega 11}\right) \cap\left(k_{\omega 12}, \infty\right) \text {. }
$$

(2) The second case is when $a<0$, one gets $-m_{n}^{\alpha}+k_{4}<0$, $b>0$, and $c$ could be positive or negative. Ignore the sign of $c$, and the intersections of $f\left(k_{\omega}\right)$ and the $x$ axis are given by

$$
\left\{\begin{array}{l}
k_{\omega 21}=\frac{-b+\sqrt{b^{2}-4 a c}}{2 a} \\
k_{\omega 22}=\frac{-b-\sqrt{b^{2}-4 a c}}{2 a} .
\end{array}\right.
$$

Thus, only when $k_{\omega 21}<k_{\omega}<k_{\omega 22}$, one gets $f\left(k_{\omega}\right)>0$. The sufficient and necessary condition for the coning motion stability can be derived as

$$
k_{\omega} \in\left(0,-\frac{m_{n}^{\omega}+k_{5}}{m_{n}^{\delta}}\right) \cap\left(k_{\omega 21}, k_{\omega 22}\right) .
$$

5.2. Fast Spinning Rate Case. For fast spinning missiles, the main factor for generation of angle of attack is the deviation of the principal axis of inertia. For the convenience of analyzing, assume that moving masses are installed at the center of mass of the projectile body, that is, $l=0$. Then, one gets $k_{2}=0$ and $k_{4}=0$. By neglecting the effect of Magnus moment and considering $k_{5}$ to be small, equation (32) can be simplified as

$$
\dddot{\xi}+\left(a_{01}+i a_{11}\right) \ddot{\xi}+\left(a_{02}+i a_{12}\right) \dot{\xi}+i a_{13} \xi=0,
$$

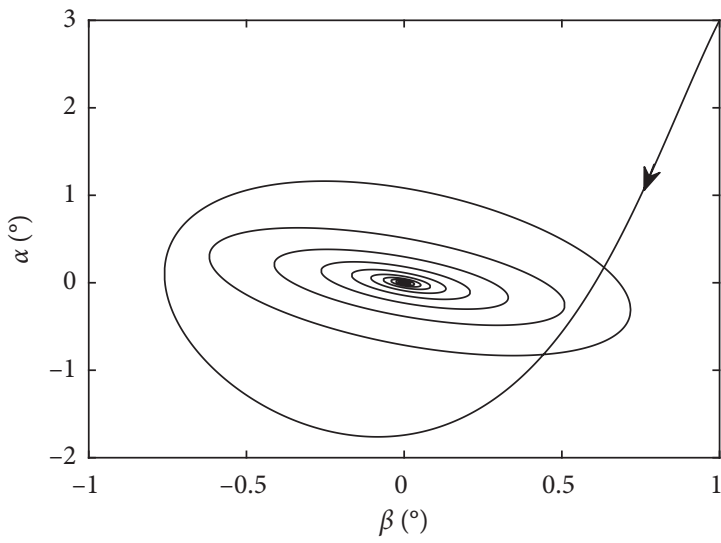

(a)

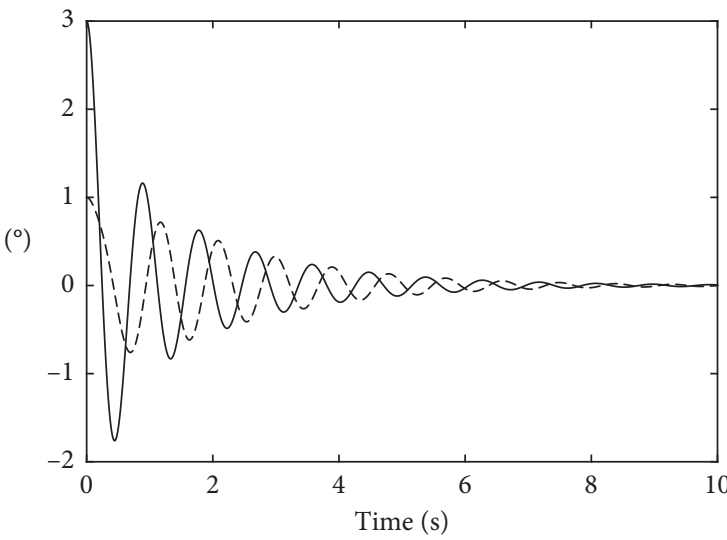

$\alpha$
$--\beta$

(b)

FIgURE 3: Simulation results for $k_{\omega}=0.1933$. (a) The stable coning motion. (b) Curves of angle of attack and angle of sideslip.

where $a_{01}=-m_{n}^{\omega}, \quad a_{11}=\left(k_{1} k_{3} k_{\omega}-1\right) /\left(k_{3} k_{\omega}\right), \quad a_{02}=k_{1} /$ $\left(k_{3} k_{\omega}\right), a_{12}=\left(k_{\omega} m_{n}^{\delta}+m_{n}^{\omega}\right) /\left(k_{3} k_{\omega}\right)$, and $a_{13}=-m_{n}^{\alpha} /\left(k_{3} k_{\omega}\right)$.

The characteristic equation of equation (46) is given by

$$
\lambda^{3}+\left(a_{01}+i a_{11}\right) \lambda^{2}+\left(a_{02}+i a_{12}\right) \lambda+i a_{13}=0 .
$$

According to the theorem proved in [18], the sufficient and necessary condition for stability of the moving mass missile under fast spinning rate with rate loops can be expressed as

$$
\left\{\begin{array}{l}
a_{01}>0, \\
a_{01}^{2} a_{02}+a_{01} a_{11} a_{12}-a_{12}^{2}>0, \\
\left(a_{01}^{2} a_{02}+a_{01} a_{11} a_{12}-a_{12}^{2}\right)\left(a_{01} a_{12} a_{13}\right)-\left(a_{01}^{2} a_{13}\right)^{2}>0 .
\end{array}\right.
$$

Because $a_{01}=-m_{n}^{\omega}>0$, equation (48) is rewritten as

$\left\{\begin{array}{l}c_{1}=a_{01}^{2} a_{02}+a_{01} a_{11} a_{12}-a_{12}^{2}>0, \\ c_{2}=a_{01} a_{12} a_{13}>0, \\ c_{3}=\left(a_{01}^{2} a_{02}+a_{01} a_{11} a_{12}-a_{12}^{2}\right)\left(a_{01} a_{12} a_{13}\right)-\left(a_{01}^{2} a_{13}\right)^{2}>0 .\end{array}\right.$ 
Substituting all the coefficients into equation (49) yields

$$
\begin{aligned}
& c_{1}=a_{01}^{2} a_{02}+a_{01} a_{11} a_{12}-a_{12}^{2} \\
& =\frac{-m_{n}^{\omega} m_{n}^{\delta} k_{\omega}-\left(m_{n}^{\omega} m_{n}^{\delta} k_{1} k_{3}+\left(m_{n}^{\delta}\right)^{2}\right) k_{\omega}^{2}}{k_{3}^{2} k_{\omega}^{2}}>0, \\
& c_{2}=a_{01} a_{12} a_{13}=\frac{-m_{n}^{\omega} m_{n}^{\alpha}\left(k_{\omega} m_{n}^{\delta}+m_{n}^{\omega}\right)}{k_{3}^{2} k_{\omega}^{2}}>0, \\
& c_{3}=-\frac{p_{1} k_{\omega}^{2}+p_{2} k_{\omega}+p_{3}}{k_{3}^{4} k_{\omega}^{3}}>0,
\end{aligned}
$$

where

$$
\begin{aligned}
& p_{1}=\left(m_{n}^{\omega}\right)^{2}\left(m_{n}^{\delta}\right)^{2} m_{n}^{\alpha} k_{1} k_{3}+m_{n}^{\omega}\left(m_{n}^{\delta}\right)^{3} m_{n}^{\alpha}, \\
& p_{2}=2\left(m_{n}^{\omega}\right)^{2}\left(m_{n}^{\delta}\right)^{2} m_{n}^{\alpha}+\left(m_{n}^{\omega}\right)^{3} m_{n}^{\delta} m_{n}^{\alpha} k_{1} k_{3}+\left(-m_{n}^{\omega}\right)^{4}\left(m_{n}^{\alpha}\right)^{2} k_{3}^{2}, \\
& p_{3}=\left(m_{n}^{\omega}\right)^{3} m_{n}^{\delta} m_{n}^{\alpha} .
\end{aligned}
$$

For the moving mass control missile under fast spinning rate, one has $\left|m_{n}^{\omega} m_{n}^{\delta} k_{1} k_{3}\right|>\left(m_{n}^{\delta}\right)^{2}$; thus, equation (50) is always true. Because $m_{n}^{\alpha}>0$, to make equation (51) true, one should have

$$
k_{\omega}<\frac{-m_{n}^{\omega}}{m_{n}^{\delta}}=k_{\omega 21} .
$$

To make equation (52) true, one should have

$$
p_{1} k_{\omega}^{2}+p_{2} k_{\omega}+p_{3}<0 \text {. }
$$

For fast spinning missile, one has $p_{1}>0, p_{2}<0$, and $p_{3}<0$. Thus, the true condition for equation (55) can be obtained as

$$
0<k_{\omega}<\frac{-p_{2}+\sqrt{p_{2}^{2}-4 p_{1} p_{3}}}{2 p_{1}}=k_{\omega 22} .
$$

Finally, the sufficient and necessary condition for stability of moving mass missile under fast spinning rate with rate loops can be expressed as

$$
k_{\omega} \in\left(0, k_{\omega 21}\right) \cap\left(0, k_{\omega 22}\right) .
$$

\section{Numerical Simulation Results}

To demonstrate the proposed stability condition above, numerical simulations are run for two sample moving mass missiles with different spinning rates.

6.1. Slow Spinning Rate Case. The parameters of a slowly spinning missile are listed in Table 1.

According to the formulae derived above, the calculated upper bound of the control loop gain is obtained as 0.3866 . The simulation results for the control loop gain $k_{\omega}=0.1933$, which satisfies the stability condition, are shown in Figure 3.
TABLe 1: Parameters of a slowly spinning missile.

\begin{tabular}{lc}
\hline Parameters & Value \\
\hline$m_{s}(\mathrm{~kg})$ & 96.6 \\
$\mu$ & 0.04 \\
$L(\mathrm{~m})$ & 1.5 \\
$S\left(\mathrm{~m}^{2}\right)$ & 0.2 \\
$\dot{\gamma}\left(\mathrm{rad} \cdot \mathrm{s}^{-1}\right)$ & 10 \\
$l(\mathrm{~m})$ & 0.1 \\
$I_{1}\left(\mathrm{~kg} \cdot \mathrm{m}^{2}\right)$ & 5.4 \\
$I_{2}\left(\mathrm{~kg} \cdot \mathrm{m}^{2}\right)$ & 58.5 \\
$C_{n}^{\omega_{z}}$ & -5.3 \\
$C_{n}^{\alpha}$ & -0.1 \\
$C_{m \alpha}$ & -1.5 \\
$V\left(\mathrm{~m} \cdot \mathrm{s}^{-1}\right)$ & 1140 \\
\hline
\end{tabular}

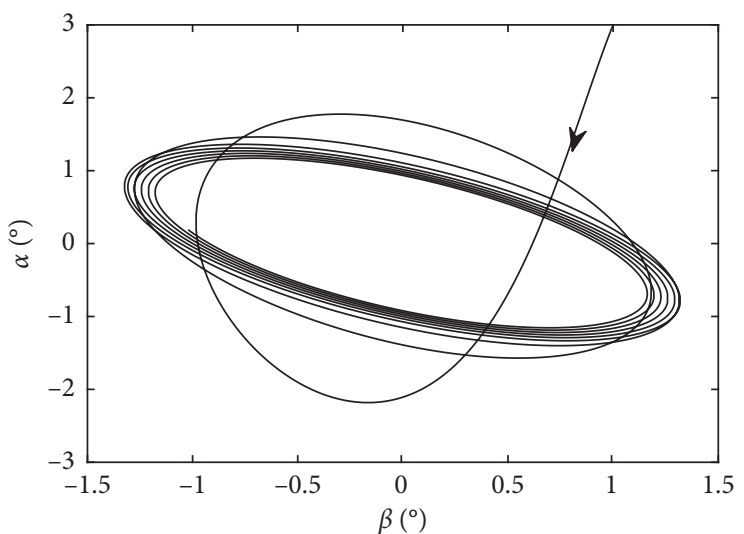

(a)

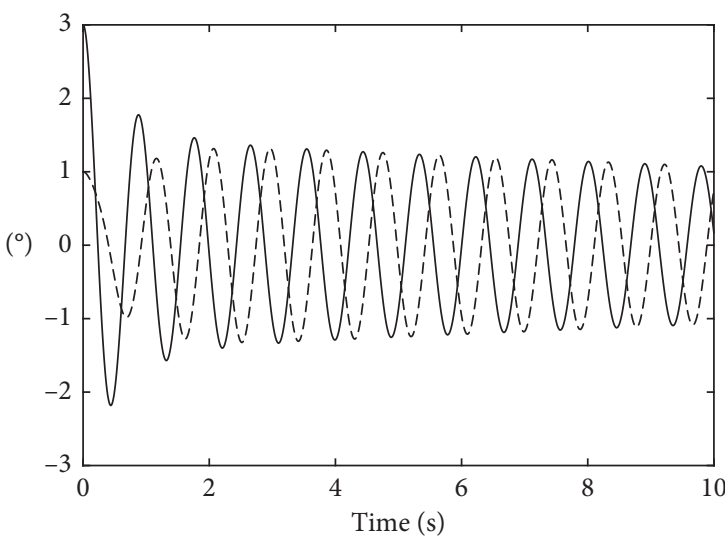

$-\alpha$

(b)

FIGURE 4: Simulation results for $k_{\omega}=0.3866$. (a) The critical coning motion. (b) Curves of angle of attack and angle of sideslip.

It can be seen obviously that the coning motion of the missile converges to zero quickly.

The simulation results for the critical control loop gain $k_{\omega}=0.3866$ are shown in Figure 4. It is observed that the coning motion of the missile neither converges nor diverges but presents a critical stable state. The simulation results for $k_{\omega}=0.5798$ are shown in Figure 5. It can be seen that the coning motion is divergent. 


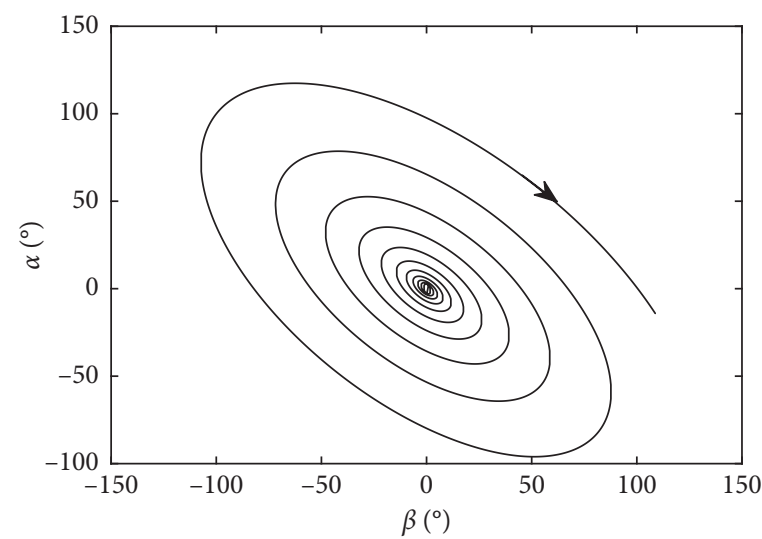

(a)

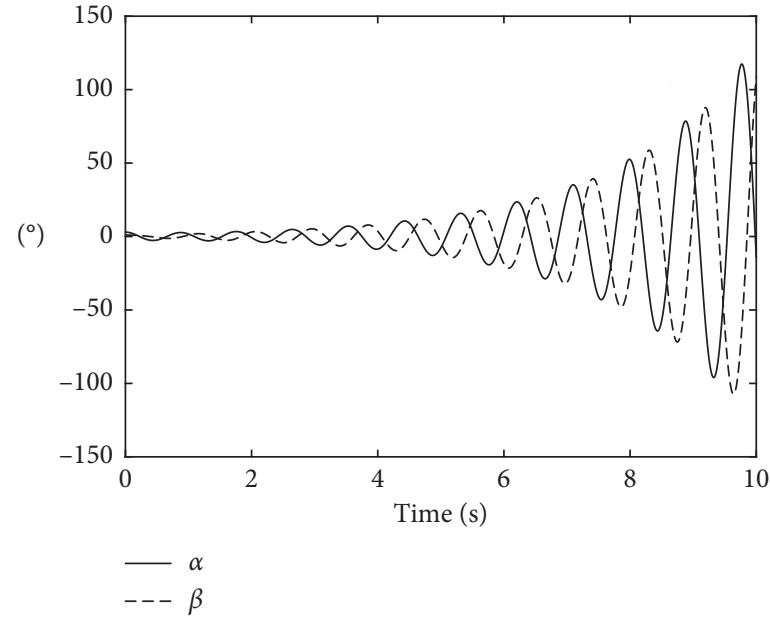

(b)

FIgURE 5: Simulation results for $k_{\omega}=0.5798$. (a) The unstable coning motion. (b) Curves of angle of attack and angle of sideslip.

TABLE 2: Upper bounds of $k_{\omega}$ under different installation positions of moving masses.

\begin{tabular}{lcccccc}
\hline Parameters & \multicolumn{5}{c}{ Value } \\
\hline$l(\mathrm{~m})$ & -0.1 & 0 & 0.1 & 0.2 & 0.3 & 0.4 \\
$k_{\omega}$ & 0.31 & 0.35 & 0.385 & 0.416 & 0.44 & 0.46 \\
\hline
\end{tabular}

TABLE 3: Upper bounds of $k_{\omega}$ under different spinning rates.

\begin{tabular}{lcccccccccc}
\hline Parameters & \multicolumn{10}{c}{ Value } \\
\hline$\dot{\gamma}(\mathrm{rad} / \mathrm{s})$ & 1 & 2 & 3 & 4 & 5 & 6 & 7 & 8 & 9 \\
$k_{\omega}$ & 0.82 & 0.77 & 0.71 & 0.67 & 0.61 & 0.56 & 0.50 & 0.45 & 0.40 & 0.35 \\
\hline
\end{tabular}

6.2. Influence of the System Parameter. In this section, the influence of the location of the moving mass $l$ and the spinning rate of the missile $\dot{\gamma}$ on the stability criterion is demonstrated. The relation between the installation position $l$ of the moving mass and the upper bound of the control loop gain $k_{\omega}$ is shown in Table 2. It can be observed from the table that the upper bound of $k_{\omega}$ increases as the location of the moving mass moves towards the warhead. This is because with the increase of $l$, the static stability of the missile is continuously strengthened, which leads to the increase of the dynamic stability region and the increase of the upper bound of $k_{\omega}$.

The relationship between the spinning rate and the upper bound of the design gain $\mathrm{kw}$ is shown in Table 3.

As can be seen obviously, the increase of the spinning rate decreases the stable region of the control design gain. This is because the higher spinning rate leads to a more serious coupling between pitch and yaw channels.

6.3. Fast Spinning Rate Case. The parameters of a fast spinning missile are listed in Table 4.

The upper bound of $k_{\omega}$ in this case is 0.5522 according to the stability condition described in equation (57). The
TABLe 4: Parameters of a slowly spinning missile.

\begin{tabular}{lc}
\hline Parameters & Value \\
\hline$m_{s}(\mathrm{~kg})$ & 96.6 \\
$\mu$ & 0.04 \\
$L(\mathrm{~m})$ & 1.5 \\
$S\left(\mathrm{~m}^{2}\right)$ & 0.15 \\
$\dot{\gamma}\left(\mathrm{rad} \cdot \mathrm{s}^{-1}\right)$ & 1000 \\
$I_{1}\left(\mathrm{~kg} \cdot \mathrm{m}^{2}\right)$ & 5.4 \\
$I_{2}\left(\mathrm{~kg} \cdot \mathrm{m}^{2}\right)$ & 58.5 \\
$C_{n}^{\omega_{z}}$ & -4.8 \\
$C_{n}^{\alpha}$ & 0.1 \\
$V\left(\mathrm{~m} \cdot \mathrm{s}^{-1}\right)$ & 1140 \\
\hline
\end{tabular}

coning motions under $k_{\omega}=0.4418$ and $k_{\omega}=0.6626$ are shown in Figures 6 and 7, respectively, from which it can be seen that the coning motion is stable when $k_{\omega}=0.4418$, while it is unstable when $k_{\omega}=0.6626$.

Furthermore, the upper bound of $k_{\omega}$ under different spinning rates is shown in Table 5 . It can be verified that the stable region of the control gain increases with the increase of the spinning rate. This is because the higher spinning rate causes a stronger inertia moment. 


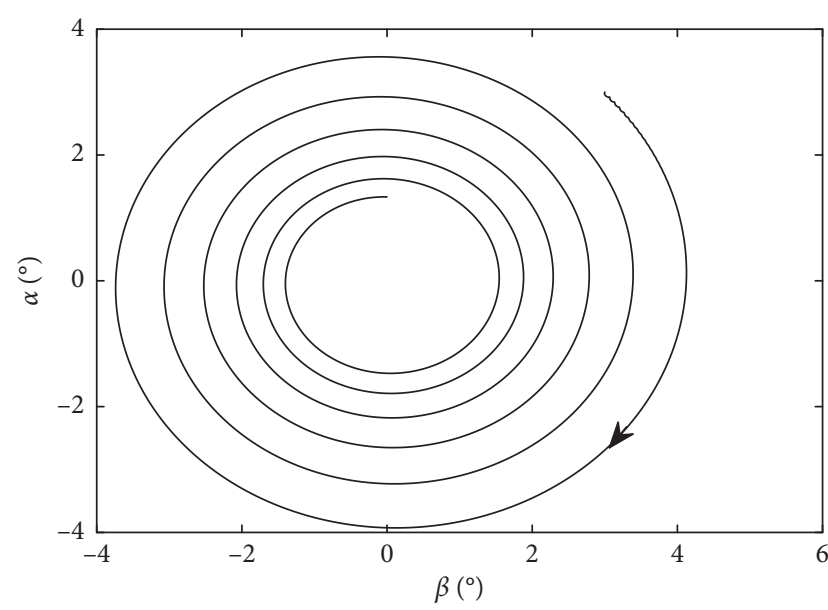

FIgURE 6: The stable coning motion.

TABLe 5: Upper bounds of $k_{\omega}$ under different spinning rates.

\begin{tabular}{llllllll}
\hline Parameters & \multicolumn{7}{c}{ Value } \\
\hline$\dot{\gamma}(\mathrm{rad} / \mathrm{s})$ & 500 & 600 & 700 & 800 & 900 & 1000 & 1100 \\
$k_{\omega}$ & 0.14 & 0.25 & 0.33 & 0.41 & 0.49 & 0.55 & 0.61 \\
\hline
\end{tabular}

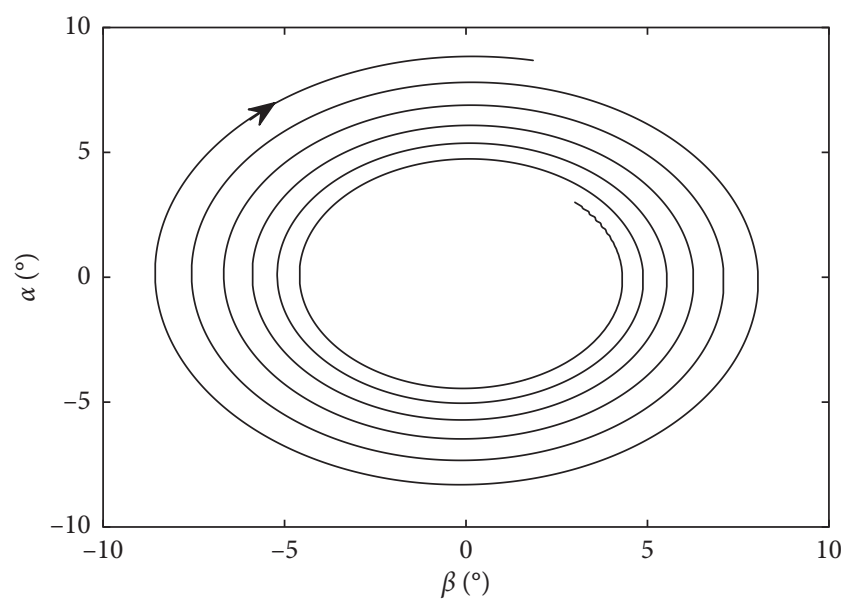

Figure 7: The unstable coning motion.

\section{Conclusion}

In this paper, the mathematical equation of a moving mass spinning missile is established. The sufficient and necessary condition of the coning motion stability for moving mass missiles with angular rate loops is analytically derived under different spinning rates and further verified by numerical simulations. Simulation results show that there exists a stability boundary value for the control gain. If the control gain exceeds it, the coning motion of the missile will diverge and the system will become unstable. It is also noticed that for the slowly spinning missile, as the location of the moving mass increases, the stability region of the system increases, while the spinning rate of the missile increases and the stability region of the system decreases greatly. For the fast spinning missile, the system stability region increases with the increase of the spinning rate. This paper is mainly based on the linearization theory of projectiles, so the stability condition obtained in this paper is applicable to the linearized missile model. In the future, we will focus on the stability analysis of nonlinear model of the moving mass control missile.

\section{Nomenclature}

$\begin{array}{ll}C x: & \text { Drag coefficient } \\ C_{y}^{\alpha}: & \text { Lift coefficient } \\ F: & \text { Force vector, } \mathrm{kg} \cdot \mathrm{m} / \mathrm{s}^{2} \\ \mathbf{H}: & \text { Angular momentum vector, } \mathrm{kg} \cdot \mathrm{m}^{2} / \mathrm{s} \\ \mathbf{I}: & \text { Inertial moment, } \mathrm{kg} \cdot \mathrm{m}^{2} \\ k_{\omega}: & \text { Gain of angular rate feedback } \\ l: & \text { Installation position of the moving mass } \\ L: & \text { Airframe diameter, } \mathrm{m} \\ \mathbf{M}: & \text { Force moment vector, } \mathrm{kg} \cdot \mathrm{m}^{2} / \mathrm{s}^{2} \\ m: & \text { Mass } \\ m_{\omega x}^{\prime}: & \text { Coefficient of roll damping moment } \\ m_{y}^{\alpha}: & \text { Coefficient of static moment } \\ m_{\omega y}^{\prime}: & \text { Coefficient of damping moment } \\ m_{\mu}: & \text { Coefficient of Magnus moment } \\ n_{y}, n_{z}: & \text { Input command } \\ Q: & \text { Dynamic pressure, } \mathrm{N} \cdot \mathrm{m}^{2} \\ \mathbf{r}_{1}, \mathbf{r}_{2}: & \text { Position vector of the moving mass } \\ S: & \text { Reference area, } \mathrm{m}^{2} \\ \mathbf{V}: & \text { Velocity vector } \\ \alpha: & \text { Angle of attack } \\ \beta: & \text { Angle of sideslip } \\ \vartheta, \psi, \gamma: & \text { Pitch, yaw, and roll angle, rad }\end{array}$

$\delta_{y}, \delta_{z}$ : Radial displacement of the moving mass

$\delta_{y c}, \delta_{z c}$ : Radial displacement command of the moving mass

$\mu: \quad$ Mass ratio

$\omega$ : Angular rate vector

Subscripts

$B$ : Missile body

S: Missile system.

\section{Data Availability}

The data used to support the findings of this study are available from the corresponding author upon request.

\section{Conflicts of Interest}

The authors declare that they have no conflicts of interest.

\section{References}

[1] R. D. I. Robinett, B. R. Sturgis, and S. A. Kerr, "Moving mass trim control for aerospace vehicles," Journal of Guidance, Control, and Dynamics, vol. 19, no. 5, pp. 1064-1070, 1996.

[2] B. Raymond, S. Beverly, and R. Rush, "A moving mass trim control system for reentry vehicle guidance," in Proceedings of the AIAA Atmospheric Flight Mechanics Conference, AIAA, pp. 644-650, San Diego, CA, USA, July 1996.

[3] T. Petsopoulos, F. J. Regan, and J. Barlow, "Moving-mass roll control system for fixed-trim re-entry vehicle," Journal of Spacecraft and Rockets, vol. 33, no. 1, pp. 54-60, 1996.

[4] G. Changsheng, J. Wuxing, and W. Pengxin, "Research on application of single moving mass in the reentry warhead 
maneuver," Aerospace Science and Technology, vol. 30, no. 1, pp. 108-118, 2013.

[5] W. Songyan, Y. Ming, and W. Zicai, "Moving-mass control system for spinning vehicle guidance based on GA," in Proceedings of the 1st International Symposium on Systems and Control in Aerospace and Astronautics, Harbin, China, January 2006.

[6] Z. Xiaoyu, Z. Yuxin, X. Dexin, and H. Kunpeng, "Sliding mode control for mass moment aerospace vehicles using dynamic inversion approach," Mathematical Problems in Engineering, vol. 2013, Article ID 284869, 11 pages, 2013.

[7] Z. Xiaoyu, L. Peng, X. Dexin, M. Ben, and H. Kunpeng, "Nonlinear predictive control of mass moment aerospace vehicles based on ant colony genetic algorithm optimization," Mathematical Problems in Engineering, vol. 2013, Article ID 858190, 10 pages, 2013.

[8] C. Murphy, "Angular motion of a re-entering symmetric missile," Astrodynamics Guidance and Control Conference, vol. 3, no. 7, pp. 1275-1282, 1964.

[9] C. H. Murphy, "Angular motion of spinning almost-symmetric missiles," Journal of Guidance and Control, vol. 2, no. 6, pp. 504-510, 1979.

[10] D. H. Flatus, "Angle-of-attack control of spinning missiles," Journal of Spacecraft and Rockets, vol. 12, no. 4, pp. 228-234, 1975.

[11] Y. Xiaoyong, Y. Shuxing, and Z. Cheng, "Coning motion of spinning missiles induced by the rate loop," Journal of Guidance, Control, and Dynamics, vol. 33, no. 5, pp. 14901499, 2010.

[12] L. Keyong, Y. Shuxing, and Z. Liangyu, "Stability of spinning missiles with an acceleration autopilot," Journal of Guidance, Control, and Dynamics, vol. 35, no. 3, pp. 774-786, 2012.

[13] Z. Wei, Y. Shuxing, and D. Jinlong, "Coning motion instability of spinning missiles induced by hinge moment," Aerospace Science and Technology, vol. 30, no. 1, pp. 239-245, 2013.

[14] G. F. Carrier and J. W. Miles, "On the annular damper for a freely precessing gyroscope," Journal of Applied Mechanics, vol. 27, no. 2, pp. 237-240, 1960.

[15] A. El-Gohary, "On the control of programmed motion of satellite-gyrostat containing fluid," Mechanics Research Communications, vol. 26, no. 3, pp. 269-275, 1999.

[16] A. El-Gohary, "On the control of programmed motion of a rigid body containing moving masses," International Journal of Non-linear Mechanics, vol. 35, no. 1, pp. 27-35, 2000.

[17] E. L. Clark Jr and A. E. Hodapp Jr, "Effects of products of inertia on re-entry vehicle roll behavior," Journal of Spacecraft and Rockets, vol. 8, no. 2, pp. 155-161, 1971.

[18] E. Frank, "On the zeros of polynomials with complex coefficients," Bulletin of the American Mathematical Society, vol. 52, no. 2, pp. 144-158, 1946. 


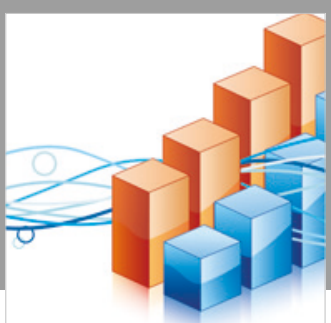

Advances in

Operations Research

\section{-n-m}
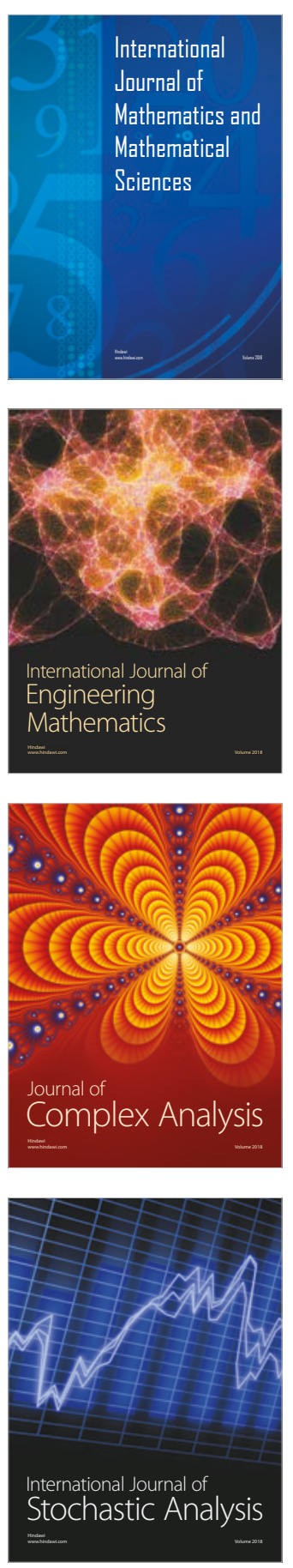
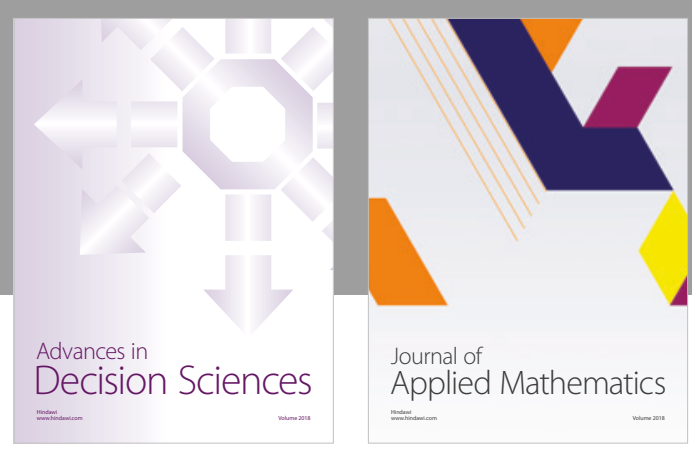

Journal of

Applied Mathematics
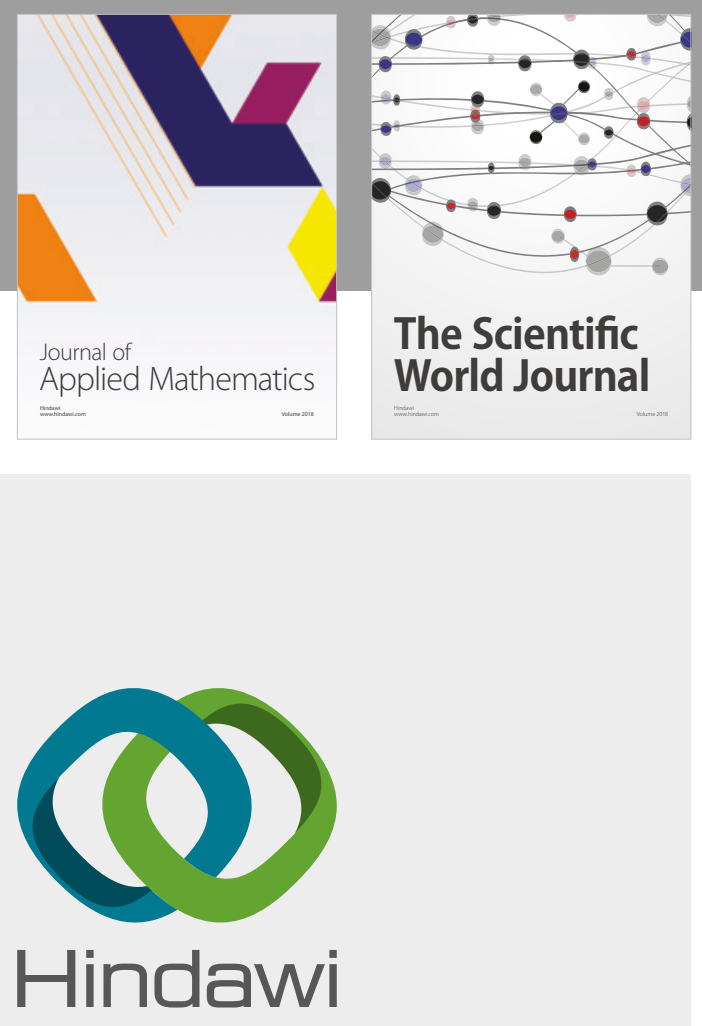

Submit your manuscripts at

www.hindawi.com

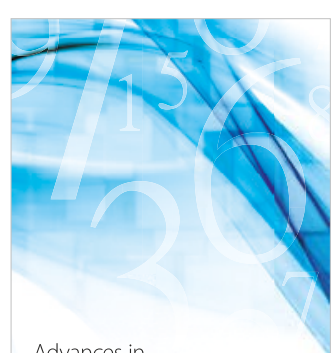

Advances in
Numerical Analysis
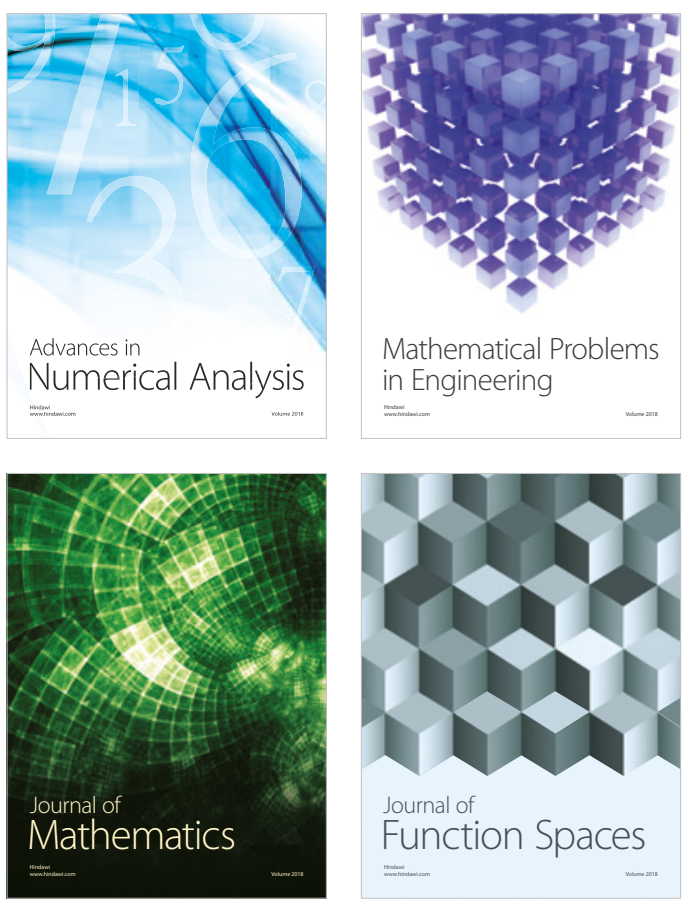

Mathematical Problems in Engineering

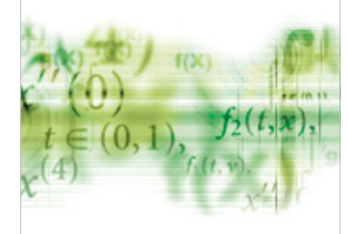

International Journal of

Differential Equations

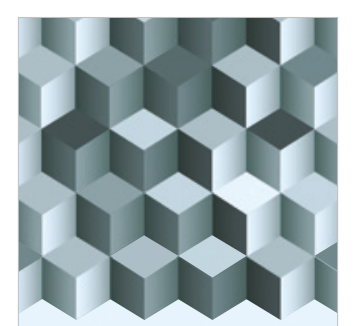

Journal of

Function Spaces

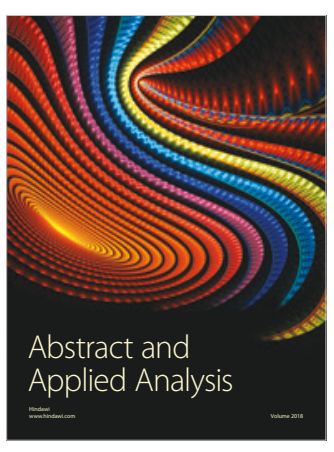

The Scientific

World Journal

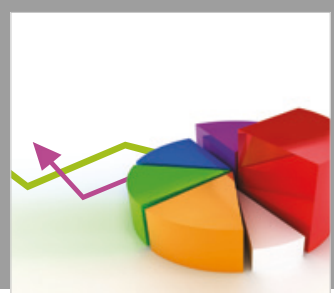

Journal of

Probability and Statistics
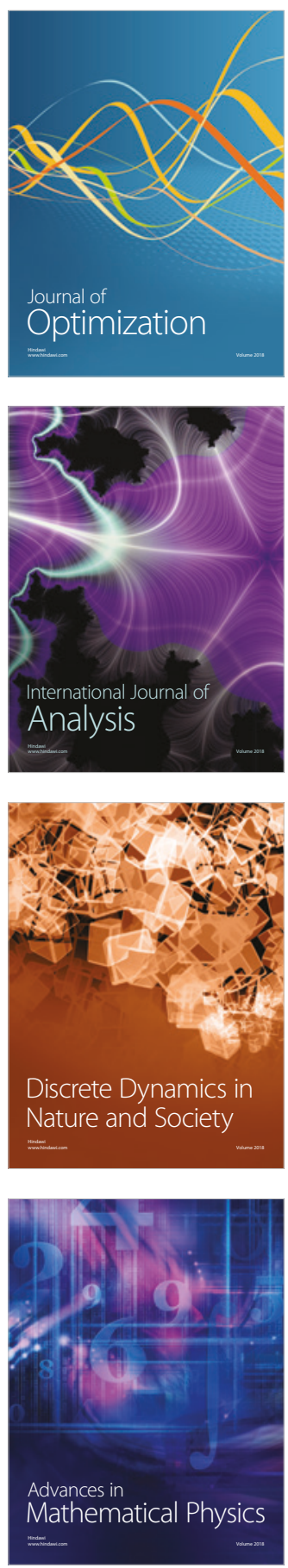